\title{
CLINICAL AND NEUROLOGICAL PECULIARITIES OF LYME DISEASE
}

\author{
Tetiana Malysh \\ Postgraduate Student, Shupik National Healthcare University of Ukraine, Ukraine \\ e-mail: dr.tatiana.malish@gmail.com, orcid.org/0000-0002-6930-6521
}

\section{Summary}

Goals of our research work are to analyze frequency and prevalence of Lyme borreliosis (LB), to understand engagement of nervs system in pathological process. Also to assess clinical Lyme disease (LD) stages, features, diagnosis, treatment and prevention methods based on the collected data and literature. The data on diagnosis, treatment, prevention of $\mathrm{LD}$ and neuroborreliosis (LNB) are carefully gathered from international and domestic publications at PubMed over 2015-2020 years. LB has stages in the course of the disease. The clinical manifestations of this disease are different. LD affects the central and peripheral nervs system, has a multiorgan character, possibly an acute and chronic course of the disease. At the moment, there are various methods for the diagnosis, treatment and prevention of LB, but pathogenesis of the disease has not been sufficiently studied, methods of vaccination have not yet been developed. Damage of nervs system in chronic LD requires further study of the pathogenetic mechanisms and factors of the development of this pathology.

Keywords: Lyme borreliosis, neuroborreliosis, serological tests, neurological features, migratory erythema.

\section{DOI: https://doi.org/10.23856/4432}

\section{Introduction}

The first Lyme disease (LD) case were identified in Ukraine in 1994. Lyme disease (LD) official records started only since 2000. Nowadays, Lyme disease (LD) cases are registered in all regions across country (Nakaz MOZ Ukrainy № 218, 2005). Lyme borreliosis (LB) morbidity in Ukraine continuously growing. It is now officially classified as an infectious disease that causes or could potentially cause significant morbidity and mortality (Centralized Information System for Infectious Diseases of the WHO Regional Office for Europe). Multicounty LD cases analysis shows that LD most frequently occur in the forest area in moderate-continental climate zone. It is possible to identify zoo-areas for certain species of ixodes mite (IM). The pathogenesis of LD had been relatively little examined. After mite bites and enters Borrelia into human skin, the pathological process begin to develop and causes interaction between numerous factors, peculiar and non-peculiar resistance of macroorganisms (Wackernagel et al., 2005). Nowadays, the tick and mite-borne borreliosis that characterized by a predominant lesion of the nervs system, named "neuroborreliosis" (Zinchuk, 2010). Frequency and severity of lesions of the nervs system depend on LD patients' place of residence - on average it ranges from $11-40 \% \%$. For example, in Central and North America it is about $11 \%$ (Centralized Information System for Infectious Diseases of the WHO Regional Office for Europe; Hansen et al., 1991), in the European Union - 29-33\% (Infektsiinyi biuleten pro stan infektsiinoi zakhvoriuvanosti v Ukraini, 2016; Popovych, 2006), in Asia - 16\%. The lack of classification of broad spectrum of neurological and psychosomatic disorders in its acute and chronic stages predetermined needs for this research. Deep comprehensive 
clinical, neurological and instrumental peculiarities of nervs system lesions in LB became the topic of our research.

\section{The purpose, materials and methods of the study}

Aim of our study is to analyze LB frequency and prevalence. Based on literature review we trying to understand how nervs system involved in the pathological process. We aim to assess specific features of the LD clinical conditions, diagnosis, treatment, prevention at each stage. We use carefully selected data from our review of world and domestic literature at PubMed for the period 2015-2020 years. The search criteria: "Lyme disease", "neuroborreliosis", "chronic form", "acute period", "lesions" nervs system", "laboratory diagnosis", "serological diagnosis", "cognitive disorders", "etiopathogenetic therapy", "prevention of Lyme disease". We follow European and Ukrainian guidelines on the diagnosis, treatment and prevention of LD and LNB.

\section{Etiopathogenesis of Lyme borreliosis}

LB is an infectious communicable disease caused by the spirochete Borrelia burgdorferi sensu lato and carrier is Ixodes mites. LB spreads globally, but most often causing health problems in the northern hemisphere, including Ukraine. The LD population risk group includes 30-60 years old. Percent of infected women is the highest. Children are only about $10 \%$ of the total number of LD patients (Nakaz MOZ Ukrainy № 218, 2005). Borrelia can persist in the human body for up to 10 years (Miklossy et al., 2004). The antigenic heterogeneity of these bacteria can alter antigenic structure, inhibition of complement-dependent phagocytosis. L form transformation of Borrelia into cyst maybe one of those 31 factors that ensure long-term persistence of the pathogen and evasion of the immune response and development of chronic forms (Zinchuk, 2010). The pathogenesis of LD studied unsufficiently. Pathological process developed after the suction of the mite and the entry of Borrelia into human skin, causing interaction with various factors of specific and nonspecific resistance of the macroorganism (Wackernagel et al., 2005).

LB develop in stages without clear identifier from one stage to another. LB can also mimicking a number of other diseases (Centralized Information System for Infectious Diseases of the WHO Regional Office for Europe; Zinchuk, 2010):

Stage 1 (local infection): at the tick bite place $80-90 \%$ of patients develop a local infection in the form of migrating erythema, develop flu-like symptoms, including fever, headache, arthralgia, myalgia. These symptoms can occur from a few days to weeks after infection. Migratory erythema is a reliable clinical marker of LB. At the same time, migratory erythema is reported only in approximately $25-50 \% \%$ of acute cases of LB.

Stage 2 (dissemination stage) occurs in few weeks, sometimes months. From the bite place the pathogen enters with lymph and blood into internal organs, lymph nodes and joints. It can also cause symptoms of damage of the cardiovascular and nervs systems.

Stage 3 (organs damage): may take months and years after the onset of the disease. This is a chronic form of LB when body develops persistent arthritis and chronic neurological symptoms. The chronic form has a recurrent nature with alternating exacerbations, asymptomatic and gradual progression of disease (Bush et al., 2018). The acute period of Lyme disease corresponds to a period of 1 to 3 months, sub-acute - from 3 to 6 months, chronic - when the persistence of symptoms of LB continue for more than 6 months. There is strong correlation between severity and duration of LD. Chronic infection can be in continuous and recurrent forms (Bush et al., 2018). 
Clinical manifestations of LB is multidimensional and can affect multiple organs. LB can runs with syntropic involvement in the pathological process of various patient's organs and systems, such as skin, musculoskeletal system, nervs system, heart, liver, eyes (Zinchuk, 2010). Therefore, the LD differs by stages and systemic manifestations.

\section{Clinical manifestations of Lyme borreliosis}

Central nervs system lesions in the acute period of LD. In this stage tick-borne borreliosis characterized by a predominant lesion of the nervs system, use the term "neuroborreliosis". Statistical analysis of the LD cases with a predominant lesion of the central nervous system shows that during the acute period of the disease its main forms are meningitis and meningoencephalitis. Clinical research revealed a number of key features that are common for the meningeal clinical conditions of the disease - headache of varying intensity, nausea, vomiting, photophobia, increased sensitivity to light and sound, pain when moving the eyeballs (Nakaz MOZ Ukrainy № 218, 2005).

Lesions of the peripheral nervous system in the acute period of LD. Long-term studies of LD at different stages indicate that during the acute period of the disease in most cases there are neurological manifestations of lesions of the peripheral nervous system (Bremell et al., 2011; Wang et al., 2014; Kravchuk et al., 1992; Halperin et al., 1991). The variety of the peripheral nervous system lesions during the acute period of the disease can be described by two syndromes - algic and amyotrophic (Badalyan et al., 1994; Pachner et al., 1995). Algic syndrome manifested by neuralgia, myalgia, plexalgia, polyradiculopathy (Bremell et al., 2011; Wang et al., 2014, Kravchuk et al., 1992). Amyotrophic syndrome accompanied by segmental radiculoneuritis, sporadic facial nerve neuritis, mononeuritis in the area of bite, limb paresis, radiculoalgia, plexalgia, mono- and polyneuritis, radicular sensory disorders in all parts of the spine (Bremell et al., 2011; Wang et al., 2014).

An amyotrophic syndrome in LB may develop own symptoms such as Bannwart's lymphocytic meningopolyradiculoneuritis with headache, fever, radicular pain, weakness and loss of sensitivity, which develops in approximately 15\% of untreated patients (Wang et al., 2014). Neurological disorders appear after erythema is over. It manifested by pain in spinal nerves roots or cervical and thoracic spine lesions (Bremell et al., 2011). The meningial symptoms are weakly expressed or can be absent (Halperin et al., 1991).

Lesions of the central nervous system in the chronic stage of neuroborreliosis. At the chronic stage of the LD, lesions of the central and peripheral nervous system are occurred with the same frequency (Matschke et al., 1987). The central nervous system lesions can be in a form of encephalopathy, encephalitis, encephalomyelitis, cerebrovascular neuroborreliosis (Mancardi et al., 1989; Reimers et al., 1992). At a chronic stage central nervous system demage can be in the form cerebral vasculitis (cerebrovascular neuroborreliosis). It takes 3-7 months from the beginning of the disease (Krupp et al., 1991; Kortela et al., 2017). This pathology carecterised by moderate manifestations of meningeal and progressive psycho-organic syndromes (memory impairment, personality change), periodic ischemic disorders in various vascular associated areas, thromboembolism of the main vessels with the development of ischemic strokes or subarachnoid hemorrhage (Kuntzer et al., 1991).

Lesions of the peripheral nervs system in the chronic conditions of neuroborreliosis. Lesions of the peripheral nervous system in chronic LNB are mainly chronic radiculoneuropathy, polyneuropathy (Bremell et al., 2011). Months or even years after the onset of the disease, some patients may develop chronic polyradiculoneuropathy with sensory symptoms or 
radicular pain. The pathology of chronic radiculoneuropathy reveals axonal degeneration with perivascular mononuclear infiltration (Wang et al., 2014; Kravchuk et al., 1992).

At the same time pains often observed in cervical, thoracic, lumbosacral area of a spine, radicular pains, paresthesias of extremities, luck of sensitivity both symmetrical, and unilateral (Badalyan et al., 1994; Pachner et al., 1995). Muscle atrophy can be in the areas of innervation of the affected nerves (Halperin et al., 1991). Patients with chronic radiculoneuropathy often complain of radicular pain or distal paraesthesia in the extremities, less often - pain in the torso. Sometimes these manifestations are accompanied by muscle weakness (Pachner et al., 1995; Halperin et al., 1991).

\section{Modern methods of diagnosing Lyme disease}

A combination of clinical and epidemiological and laboratory results is recommended for the diagnosis of LNB. Patients with pre-existing migratory erythema and epidemiological risk may be diagnosed with acute LNB without laboratory tests. Laboratory tests are needed to confirm the diagnosis for all other patients, but their correct interpretation depends on the symptoms and duration of the disease.

However, a causal relationship between neurological symptoms and borreliosis infection cannot be easily established in patients with polyneuropathy / polyneuritis whose blood tests are positive for Borrelia (Lantos, 2015), as Borrelia-specific antibodies are detected in approximately $5-20 \%$ of healthy people. People depend on the endemicity of the region and age group (Izquierdo et al., 1992).

Therefore, the causal relationship between neurological symptoms and borreliosis infection cannot be easily established for patients with polyneurosis. Thus, the main symptoms of LNB are radicular pain, paresis of the extremities and facial nerve, meningitis. However, there are a large number of neurological symptoms that are less common and can be attributed to borreliosis infection only by appropriate studies of cerebrospinal fluid (CSF) (Johnson et al., 2014).

Antil now detection of antibodies to B. burgdorferi in CSF in patients with LB with signs of nervous system damage remains uncertain. The appearance of B. burgdorferi in CSF stimulate intrathecal immune response usually after 2 weeks of neurological manifestations. According to many authors, is one of the major diagnostic criteria for LNB (Izquierdo et al., 1992).

There is another point of view that the absence of pleocytosis and intrathecal synthesis of antibodies to B. burgdorferi does not exclude LNB even in the long term (Johnson et al., 2014). E. Roaldsnes et al. argue that the likelihood of LNB is low in the absence of typical symptoms of the disease, even when anti-Borrelia antibodies are detected in the serum.

Recently, the concentration of chemokine CXCL13 in the cerebrospinal fluid has been introduced aa a new diagnostic tool for LDL. It is used to complement antibody-based diagnostic methods. Other cytokines have also been analyzed as diagnostic markers. According to the recommendations of the European Federation of Neurological Societies (EFNS), the following 3 criteria should be considered for a reliable LNB testing, and 2 of them - for a probable LNB (Cameron et al., 2016):

- neurological symptoms;

- pleocytosis CSF (cerebrospinal fluid);

- Borrelia-specific antibodies produced intrathecally. Polymerase chain reaction (PCR) may be confirmatory if the duration of symptoms is $<6$ weeks, when antibodies to Borrelia may be absent. 
Otherwise, PCR is not recommended. There is also insufficient evidence to recommend the following tests for diagnostic purposes: microscopic analysis, chemokine CXCL 13, detection of antigens, immune complexes, lymphocyte transformation test, cyst formation, lymphocyte markers (Cameron et al., 2016).

The use of such tests as seeding, PCR or detection of B. burgdorferi antigens is limited. As of today, there is no serological test that can distinguish between past and active LB disease. PCR sensitivity is $25-30 \%$ in early LB, and $10 \%$ in chronic LNB. The main disadvantage of PCR is the large number of false-negative results (Cameron et al., 2016). Serological methods are based on the determination of a specific immune response to B. burgdorferi antigens. The most commonly used are the indirect immunofluorescence test (IDIF), enzymelinked immunosorbent assay (ELISA), and immunoblot (Western-blot) (Kaya et al., 2008; Spirin et al., 2012).

A two-level antibody test, consisting of ELISA or RIF analysis followed by immunoblotting are available. It is widely used despite significant limitations in sensitivity, especially in early infection (30-40\%) and in the convalescence phase after treatment with early LB antibiotics. A combination of clinical and epidemiological and laboratory research methods is effective in the LNB diagnosis.

\section{Preventive and treatment strategies for patients with neuroborreliosis}

To prevent LB, it is very important to remove ticks before they disappear. The risk of transmission of Borrelia increases with increasing bite time of the mite. Transmission does not occur immediately when ticks attach to the skin. There is a delay, which may depend on what type of Borrelia infects the mite. Ticks should be removed immediately with tweezers to prevent transmission of Borrelia. If parts of the suction organ remain in the skin, they can later be removed with a needle or curettage. If the head or suction organ remains in the skin, the risk of Borrelia transmission does not increase.

The bodies of nymphs and adult mites should not be compressed when they are filled with blood to prevent possible transmission of Borrelia. After removal of the tick, the patient should be informed of the need to monitor the site of the tick bite for the next 6 weeks (Cameron et al., 2016).

Some studies have reported that the risk of infection after a tick bite can be reduced by a single prophylactic injection of $200 \mathrm{mg}$ of doxycycline (87\% efficacy) (Saccomano et al., 2018). In this case, J. Piesman et al. experimentally found that doxycycline treatment on the day of removal of a potentially infected mite protected $74 \%$ of mice. When treatment was delayed until 24 hours after removal mites, only $47 \%$ of mice were protected. Prophylactic treatment was completely ineffective $\geq 2$ days after tick removal. According to the authors, the dynamics of preventive treatment with antibiotics in mice may differ from the treatment of humans. Given the results of animal studies, the management of patients, however, should be approached with caution. The results suggest that antibiotic treatment for the prevention of LB is more effective if performed immediately after removal.

There is only a very narrow window for prophylactic treatment, which may be effective after mite removal (Saccomano et al., 2018). Due to the possible effects on the intestinal flora and the risk of developing resistance due to frequent prophylaxis. Oral prophylaxis with doxycycline is not recommended in Europe. Currently, no vaccine is approved for the prevention of LB, although vaccination with recombinant LYMErix vaccine, which reduced the number of new infections in vaccinated adults by almost 80\%. was approved in the United States in 1999. 
But it was later withdrawn from the market by the manufacturer for economic reasons. This monovalent vaccine is not suitable for Europe because it protects only against B. burgdorferi sensu stricto and not against the B. afzelii and B. garinii genotypes that are more common in Europe. A polyvalent OspA vaccine developed in Europe, but it is not approved yet (Middelveen et al., 2018).

Today, all stages of LB are treated with antibiotics. Doxycycline, penicillin G, amoxicillin and ceftriaxone are the antibiotics of choice in the treatment of LNB. In the case of early LNB, doxycycline and beta-lactam (penicillin G, ceftriaxone and cefotaxime) antibiotics are equally effective in terms of regression of neurological symptoms and are tolerated equally. According to guidelines from the European Federation of Neurological Societies, adult patients with reliable or possible acute LNB (symptom duration $<6$ months) should be offered a single 14-day course of antibiotic treatment (Mygland et al., 2018). Oral doxycycline (200 mg / day) and intravenous ceftriaxone ( $2 \mathrm{~g} /$ day) are equally effective in patients with peripheral nervous system symptoms, including meningitis. Patients with manifestations of central nervous system damage are prescribed intravenous ceftriaxone ( $2 \mathrm{~g} /$ day) for 14 days; with late LNB (duration of symptoms $>6$ months) - within 3 weeks (Mygland et al., 2018). It is reported that most patients have good results and symptoms disappear within 12 months regardless of the antibiotic used. There is evidence that doxycycline, penicillin G, ceftriaxone, and cefotaxime are effective in the treatment of European LNB.

D. Bremell and L. Dotevall evaluated the effectiveness of oral doxycycline in the treatment of LNB with central nervous system symptoms. The number of cerebrospinal fluid mononuclear cells was used as a surrogate marker of treatment outcome. The study included 26 patients with central nervous system damage and 115 patients with symptoms of peripheral nervous system damage. Treatment with oral doxycycline led to a similar decrease in the number of mononuclear cells in the cerebrospinal fluid of patients with LNB and symptoms of central nervous system lesions compared with patients with LNB with symptoms of peripheral nervous system damage. All patients with central nervous system lesions showed clinical improvement after treatment, although $62 \%$ had residual symptoms at the end of follow-up. The results indicate that oral doxycycline is an effective treatment for LNB, regardless of the severity of symptoms (Bremell et al., 2014).

R. Dersch et al. found a statistically significant difference between doxycycline and beta-lactam antibiotics in terms of residual neurological symptoms after 4-12 months of treatment in a systematic review of 16 studies. Significantly fewer neurological symptoms were observed after taking cefotaxime compared to penicillin. At the same time, side effects were significantly lower when prescribing penicillin (Dersch et al., 2016).

$\mathrm{S}$. Rauer et al. argue that in the treatment of early LDL, oral doxycycline is well tolerated and its effectiveness is equivalent to the effectiveness of intravenous beta-lactam antibiotics (penicillin G, ceftriaxone and cefotaxime). In this case, 14 days is enough for the treatment of early, and 14-21 days - for the treatment of chronic LNB. Prolonged administration of antibiotics for many weeks or even months in chronic LNB with nonspecific symptoms has no additional benefit and creates a risk of serious side effects (Rauer et al., 2018).

According to A. Krause and V. Fingerle, early Lyme disease is almost always cured by a single course of antibiotics, which also prevents further disease progression. After antibiotic therapy of late manifestations of LNB, the symptoms disappear slowly, and remission is usually achieved in weeks or even months (Krause et al., 2009). Combination antimicrobial therapy or courses of antibiotics longer than 4 weeks in chronic LNB are not recommended (Krause et al., 2009). 
Thus, early antimicrobial treatment is effective in most cases of LNB and most patients return to their pre-morbid health after the recommended treatment. However, despite targeted antibiotic therapy, persistent forms of LNB with manifestations of so-called "subjective" symptoms that can last for a significant period of time after treatment (for at least 6 months).

Thus, U. Koedel and H. W. Pfister emphasize the existence of nonspecific symptoms of LDL that remain despite conventional therapy: a syndrome called post treatment Lyme disease syndrome (PTLDS). IDSA has provided a formal definition of PTLDS, but its pathogenesis and even its existence need further elucidation. PTLDS is not associated with a specific genotype of B. burgdorferi. The authors note that these patients in most cases do not suffer from persistent B. burgdorferi infection and do not benefit from additional antibiotic therapy (Koedel et al., 2017). There are currently no FDA-approved treatments for PTLDS. The safety and efficacy of non-recommended and / or adjunctive treatments have not been sufficiently studied and agreed by the medical community.

Both in vitro and in vivo, it has been found that antibiotic resistance and recurrence of LB are associated with biofilm-like formations of B. burgdorferi, B. afzelii and B. garinii, allowing Borrelia spp. to resist adverse environmental conditions, and indicating the possible role of the formed biofilm in the development of various manifestations of LB, including LNB. The prevention of LB is the use of personal protective equipment when visiting natural foci. It is important to remove ticks as early as possible. In some Studies have reported a reduction in infection through emergency prevention. Vaccination methods have not been definitively developed. The main treatment for LB is antibacterial therapy.

\section{Conclusions}

The question of pathogenetic mechanisms and risk factors for the development of pathology of the nervous system in chronic LB remains open. Nervous system compromised in LB is an urgent issue that requires improvement of methods of early diagnosis, treatment and prevention. The analysis of the literature suggests that a comprehensive research of nervous system damage at all stages of LB, urge the needs for further research.

\section{References}

Badalyan, L.O., Kravchuk, L.N., \& Sergovskaya, V.D. (1994). Nevrologicheskie sindromy pri bolezni Lajma u detej [Neurological syndromes in Lyme disease in children]. Zhurnal nevropatologii i psihiatrii - Journal of Neuropathology and Psychiatry, 32, 3-6 [in Russian].

Bremell, D., \& Dotevall, L. (2014). Oral doxycycline for Lyme neuroborreliosis with symptoms of encephalitis, myelitis, vasculitis or intracranial hypertension. European journal of neurology, 21(9), 1162-1167.

Bremell, D., \& Hagberg, L. (2011). Clinical characteristics and cerebrospinal fluid parameters in patients with peripheral facial palsy caused by Lyme neuroborreliosis compared with facial palsy of unknown origin (Bell's palsy). BMC Infectious Diseases, 11, 215-225.

Bush, L.M., \& Vazquez-Pertejo, M.T. (2018). Tick borne illness-Lyme disease. Disease-a-Month Journal, 64 (5), 195-212.

Cameron, D. J., Johnson, L.B., \& Maloney, E.L. (2016). Evidence assessments and guideline recommendations in Lyme disease: the clinical management of known tick bites, erythema migrans rashes and persistent disease. Expert review of anti-infective therapy, 12(9), 1103-1135. 
Centralized Information System for Infectious Diseases of the WHO Regional Office for Europe [Text]. (n.d.). data.euro.who.int/cisid. Retrieved from http://data.euro.who.int/cisid.

Dersch, R., Sommer, H., Rauer, S., \& Meerpohl, J. (2016). Prevalence and spectrum of residual symptoms in Lyme neuroborreliosis after pharmacological treatment: a systematic review. Journal of neurology, 263(1), 17-24.

Halperin, J.J., Volkman, D.J., \& Wu, P. (1991). Central Nervos system abnormalities in Lyme neuroborreliosis. Neurology, 41(10), 1571-1582.

Hansen, K., \& Lebech, A.M. (1991). Lyme neuroborreliosis: a new sensitive diagnostic assay for intrathecal synthesis of Borrelia burgdorferi--specific immunoglobulin $G, A$, and M. Annals of Neurology, 30 (2), 197-205.

Infektsiinyi biuleten pro stan infektsiinoi zakhvoriuvanostiv Ukraini [Infectious bulletin on the state of infectious diseases in Ukraine]. (2016). www.dsesu.gov.ua. Retrieved from http://www. dsesu.gov.ua [in Ukrainian].

Izquierdo, G., \& Aguilar, J. (1992). Validity of positive anti-Borrelia antibodies in patients with neuroborreliosis. Neurologia, 7(7), 198- 199.

Johnson, B. J., Pilgar, M.A., \& Russel, T.M. (2014). Assessment of new culture method for detection of Borrelia species from serum of lyme disease patients. Journal of clinical microbiology, 52(3), 721-724.

Kaya, A.D., Parlak, A.H., Ozturk, C.E., \& Behcet, M. (2008). Seroprevalence of Borrelia burgdorferi infection among forestry workers and farmers in Duzce, north-western Turkey. The new microbiologica, 31(2), 203-209.

Koedel, U., \& Pfister, H. (2017). Lyme neuroborreliosis. Current opinion in infectious diseases, 30(1), 101-107.

Kortela, E., Hytönen, J., Numminen, J., Overmyer, M., Saxen, H., \& Oksi J. (2017). Cerebral vasculitis and intracranial multiple aneurysms in a child with Lyme neuroborreliosis. JMM Case Reports, 4, 217-230.

Krause, A., \& Fingerle, V. (2009). [Lyme borreliosis] [Article in German]. Zeitschrift für Rheumatologie, 68(3), 239-252.

Kravchuk, L.N., Kuvshinov, Yu.V., \& Sergovskaya, V.D. (1992). Seroznye meningity pri borrelioze Lajma [Serous meningitis in Lyme borreliosis]. Zhurnal nevropatologii i psihiatrii Journal of Neuropathology and Psychiatry, 92, 41-44 [in Russian].

Krupp, L.B., Masur, D., Schwartz, J., Coyle, P.K, Langenbach, L.J., Fernquist, S.K., et al. (1991). Cognitive functioning in late Lyme borreliosis. Archives of neurology, 48(11), 1125-1129.

Kuntzer, T., Bogousslavsky, J., Miklossy, J., Steck, A.J., Janzer, R., \& Regli, F. (1991). Borrelia rhombencephalomyelopathy. Archives of neurology, 48(8), 832-836.

Lantos, P.M. (2015). Chronic Lyme disease. Infectious disease clinics of North America, 29, 325-340.

Mancardi, G.L., Del Sette, M., \& Primarera, A. (1989). Borrelia burgdorferi infection and Guillain-Barre sindrome. Lancet, 2 (8669), 985-986.

Matschke, H., \& Durch, J. (1987). [Neurologic diseases transmitted by ticks] [Article in German]. Zeitschrift für die gesamte innere Medizin und ihre Grenzgebiete, 7, 192-195.

Middelveen, M.J., Sapi, E., Burke, J., Filush, K.R., Franco, A., Fesler, M.C., et al. (2018). Persistent Borrelia Infection in Patients with Ongoing Symptoms of Lyme Disease. Healthcare (Basel, Switzerland), 6(2), 33-39.

Miklossy, J., Khalili, K., Gern, L., Ericson, R., Darekar, P., Bolle, L., et al. (2004). Borrelia burgdorferi persists in the brain in chronic lyme neuroborreliosis and may be associated with Alzheimer disease. Journal of Alzheimer's disease, 6, 639-649. 
Mygland, A., Ljøstad, U., Fingerle, V., Rupprecht, T., Schmutzhard, E., Steiner, I., et al. (2018). EFNS guidelines on the diagnosis and management of European Lyme neuroborreliosis. European journal of neurology, 17(1), 8-16.

Nakaz MOZ Ukrainy № 218 "Pro posylennia zakhodiv diahnostyky ta profilaktyky iksodovykh klishchovykh borelioziv v Ukraini» [Order of the Ministry of Health of Ukraine № 218 "On strengthening measures for diagnosis and prevention of Ixodes tick-borne borreliosis in Ukraine»]. (2005). Kyiv [in Ukrainian].

Pachner, A.R., \& Steere, A.C. (1995). The triad of neurologic mani-festations of Lyme disease: meningitis, cranial neuritis and radiculoneuritis. Journal of Neurology, 35, 47-53.

Popovych, O.O. (2006). Laim-borelioz: suchasna problema infektolohii [Lyme disease: modern problems of infectology]. Aktualna infektolohiia - Topical infectology, 3, 114-122 [in Ukrainian].

Rauer, S., Kastenbauer, S., Fingerle, V., Hunfeld, K., Huppertz, H., \& Dersch, R. (2018). Lyme Neuroborreliosis. Deutsches Ärzteblatt international, 115(45), 751-756.

Reimers, C.D., Neubert, U., Kristoferitsch, W., \& Mayr, W.R. (1992). Borrelia burgdorferi infection in Europe: an HLA-related disease? Infection, 20(4), 197-200.

Saccomano, S.J., \& Hrelic, D.A. (2018). Recognizing and treating Lyme disease. The Nurse practitioner, 43(8), 13-21.

Spirin, N.N., Baranova, N.S., Fadeeva, O.A., \& Shipova, E.G. (2012). Algoritm differencialnoj diagnostiki rasseyannogo skleroza i hronicheskogo borrelioznogo encefalomielita [Algorithm for differential diagnosis of multiple sclerosis and chronic borreliosis encephalomyelitis]. Nevrologicheskij zhurnal-Neurological journal, 4, 31-36 [in Russian].

Wackernagel, A., Bergmann, A. R. \& Aberer, E. (2005). Acute exacerbation of systemic scleroderma in Borrelia burgdorferi infection. The Journal of the European Academy of Dermatology and Venereology, 19, 93-96.

Wang, E., Shirvalkar, P.R. \& Maciel, C.B. (2014). American neuroborreliosis presenting as cranial polyneuritis and radiculoneuritis. Neurology Neuroimmunology \& Neuroinflammation, 1(3), 420-423.

Zinchuk, O.M. (2010). Laim-borelioz: kliniko-imunopatohenetychni osoblyvosti ta ekstrene preventyvne likuvannia [Lyme borreliosis: clinical and immunopathogenetic features and emergency preventive treatment]. Extended abstract of Doctor's thesis. Kyiv [in Ukrainian]. 